\title{
ON DYNAMICAL NON-STATIONARITY OF THE GALAXY SYSTEMS
}

\author{
J.P. Anosova*, L.G. Kiseleva', and V.V. Orlov \\ *Astronomical Observatory, St. Petersburg University, Russia \\ tDept. of Applied Mathematics, Herzen Pedagogical Institute, \\ St. Petersburg, Russia
}

\section{ABSTRACT}

By the computer simulations we have studied a behaviour of the virial coefficient $k(t)$ for the triples and quintets of galaxies as a function of time. In a number of cases some qua siperiodical oscillations $k(t)$ have been observed; these variations are resulted from an existence of the temporary binary aubsystems. Strong negative correlation between $k$ and $r_{m i n}\left(r_{m i n}\right.$ is a minimum separation between the bodies) is observed. A presence of a large hidden mass $M_{n}$ distributed around a triple increases the degree of stochasticity of the process $k(t)$ and decreases the correlation between $k$ and $r_{m i n}$. The process $k(t)$ must not been considered as a random one, as the autocorrelation function does not show any decreasing of the oscillation amplitude with a grow of time.

\section{INTRODUCTION}

The small galaxy groups, containing from 3 to $10 \mathrm{memb}-$ er s, are widespread in the metagalactic field. Therefore a study of their dynamical states and possible dynamical evolutions has some cosmogonic interest.

One of the most informative characteristics of dynamical states of the small galaxy groups is a virial coefficient

$$
k=T /|u|
$$

where $T$ and $U$ are the kinetic and potential energies correspondingly. An special role of this energetic characteristics 
is that it is used for the dynamical estimates of the masses of galaxy groups (see, e.g., Heisler et al. 1985). On such1 ike estimates one a ssumes that $k=1 / 2$, that is one thinks that the virial theorem is fulfilled for every observed group in all times.

Hower, the mumerical simulations (Anosova et al.1989) have shown that the dynamical states of the galaxy groups change contimously during the evolution. Correspondingly, the quantity $k$ also varies. In the former work (Anosova et al. 1990) we have shown that a stochastic dynamical evolution is typical for the triple galaxies. Therefore, we would wit that the dependences on time of the basic dynamical parameters, in particular $k(t)$, are some random processes.

An aim of this work is to study of the behaviour of the dependence $k(t)$ as a random process and an establ ishent of its possible connections with other dynamical and configurational characteristics of the triple systems. An influence to $k(t)$ of a hidden matter distributed around a triple system is al so studied.

\section{MODELS AND METHODS}

Dynamical evoluation of the system of three and five galaxies has been studied by computer simulations (Anosova et.a1. 1989). The triple systems with equal mass components and initial zero velocities have been considered. The initial configurations have been assigned inside a region $D$ of all possible configurations of the tiple systems by the uniform scanning with a step $\Delta \xi=\Delta \eta=0.05$. For the quantuple systems a close pair with a circular orbit of radius 0.01 d ta is an average size of the tiple) has been placed in the centre of masses of a triplet.

In a number of models, a triple systen has been sinked in a distributed dark matter having a density profile

$$
\rho_{h}(R)=\left\{\begin{array}{cl}
\left(M_{h^{\prime}} / 4 \pi R_{h}\right) \cdot R^{-2}, & R \leq R_{h} \\
0, & R>R_{h^{\prime}}
\end{array}\right.
$$

where $\mathrm{M}_{\mathrm{h}}$ is a total hidden mass, placed inside a sphere with a radius $R_{h}, R$ is a distance from the centre of distribution of the hidden matter which coincides with the barycentre of a triple in $t=0$. We have ignored a reverse influence of components of the triple system to the hidden mass and a dynamical friction on it. Some possibility of merging galaxies has been taken into account, a criterion by Roos and Norman (1979) for merging has been used. 
The equations of motion of the N-body problem with a "softened" potential of interaction between the bodies have been integrated by the RK4 integrator.

\section{RESULTS}

The dynamical state of the small multiple system connected by gravitation changes in time contimously. Often some temporary binary subsystems are formed which give an essential contribution to the total energy of the $\mathrm{N}$-body system. In this connection we are interested to follow a variation $k(t)$ versus time for the isolated binaries having the different eccentricities $e$ of the orbits.

The se dependences are presented in Fig. 1 for $e=0,0.1$, $0.5,0.9$. The dependences $k(t)$ have the periodical oscillations with an amplitude $\Delta \mathrm{k}=e$ and a period equal to the one of revolution of the binary. An average for a period quantity is equal to $\bar{k}=1 / 2-e^{2} / 4$; thus the dependence $k(t)$ for the isolated binary system is a strongly periodical, non-random process.

A character of this process $k(t)$ apparently changes when we consider the triple systems. The examples in Figs. 2,3 show this. In these figures the dependences $k(t)$ are presented within the interval $t \in[0,10] \tau(\tau$ is a crossing time for the triple system). The quantity $k$ has the oscillations within the interval $k \in[0.02,0.99]$. Such oscillations in Fig. 2 have no any periodical character. In Fig. 3 we observe as a non-periodical behaviour of $k(t)$ during the early stages of evolution of the system $t \in[0,5] \tau$, as a quasiperiodical oscillations $k$ within the later interval $t \varepsilon^{\prime}[5,10] \tau$. Such $a$ type of behaviour is connected with formation of a temporary binary subsystem having a negative total energy. The strong negative correlation between $k$ and $r_{m i n}$ (a minimum separation between the components in triple system) does also confirm this: the coefficient of correlation $\rho \cong-0.9$. The dependences $r_{m i n}(t)$ are shown in the lower parts of $F i g .2,3$. In order to study a degree of determinism of the process $k(t)$ we have driven the autocorrelation functions $\gamma\left(t^{\prime}\right)$ for it. The functions $\gamma\left(t^{\prime}\right)$ for the example shown in Fig. 3 are presented in Fig. 4. One can observe in this figure an abatement of $\gamma\left(t^{\prime}\right)$ upto zero during a time of $t^{\prime}=(0.3-1) t$. However, in further the function $\gamma\left(t^{\prime}\right)$ is not nearby to zero and it has some oscillations having a significant amplitude. These oscillations may speak about a quasiperiodical behaviour $k(t)$ connected with a presence in the systems of some temporary pairs having the energy $E<0$. An analysis of spectral density $G(\omega)$ for this example has been made. The maxima of spectral density correspond to the periods multiple to the pair one. Such a behaviour is typical for the models under consideration of the 
isolated galaxy triples, that is, the process $k(t)$ is no truly random one, and it has rather quasiperiodical character.

When the multiplicity of a system increases from $N=3$ to $N=5$, a degree of randomness of the process $k(t)$ grows that is demonstrated by Fig. 5 where the motationsare the same as in Figs. 2,3.

We also study an influence to the behaviour $k(t)$ from the distributed dark mass $M_{h}$, in which the triple system was sinked initially. The quantities $\mathrm{M}_{h}$ and $R_{h}$ were the model parameters; the values are the following: $M_{h}=25,50,100,200 \mathrm{~m}$ ( $m$ is the mass of one of components of the $\operatorname{triple);~} R_{h}=5,10$, $15,20 \mathrm{~d}$. A few examples of dependences $k(t)$ and $r_{m i n}(t)$ for different $M_{h}$ on $R_{h}=10 d$ within the interval $t \epsilon(0,5) \tau$ are shown in Figs. 6-8. The initial configuration of a triple was the same for all examples: $\xi=0.1, n=0.5$. As we have ignored an reverse influence by bodies to the hidden mass, the value $k$ for a triple may be more than 1 .

The Figs, 6-8 show that a character of the process $k(t)$ varies with increasing of $\mathrm{M}_{\mathrm{h}}$ : it is more fast-variable,a degree of its randomization increases. A picture of the dependence $k(t)$ for $M_{h}=200$ is similar to the one for the 5-body systems. A sign of the correlation coefficient between $\mathrm{k}$ and $r_{m i n}$ is the same (negative) for the systems with hidden mass but its value $\rho$ in module is essentially decreasing: from $\rho=0.95$ for $M_{h}=0$ to $\rho=-0.45$ for $M_{h}=200$ on these initial conditions. If $\mathrm{Fig} 6\left(\mathrm{M}_{\mathrm{h}}=0\right)$ shows a strong correlation then already for $M_{h}=25$ (Fig. 7 ) this connection is essentially weaker, e.g.; a region of the qua siperiodical variation of $k(t)$ in Fig. $7 t \in(3,5) \tau$ does not correspond to such like behaviour of $r_{\min }$. That is in this case, a presence in the triples of the close binary subsystems as well as of the double encounters not the only factor determining the evolution. Other important factor is the hidden mass that leads to a stochastization of the dynamics of the triple systems (Anosova et al. 1990).

During the evolution of the systems in all cases under consideration the amplitude of oscillations $k(t)$ ha $s$ no tendency of decreasing (as with a dark matter or without it). So large amplitude of oscillations indicates that use of the virial theorem in order to estimate the mass of any galaxy system under study may lead to a possible error in a factor 10 and more even without taking into account some effects of projection. 


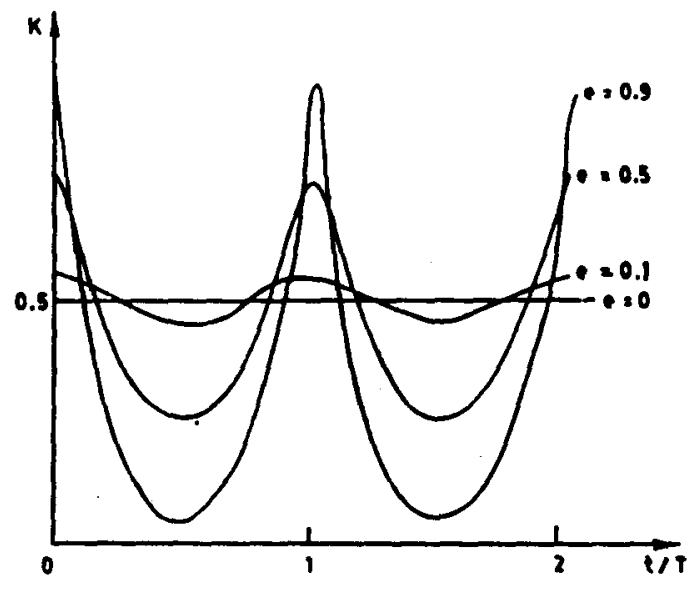

Figure 1

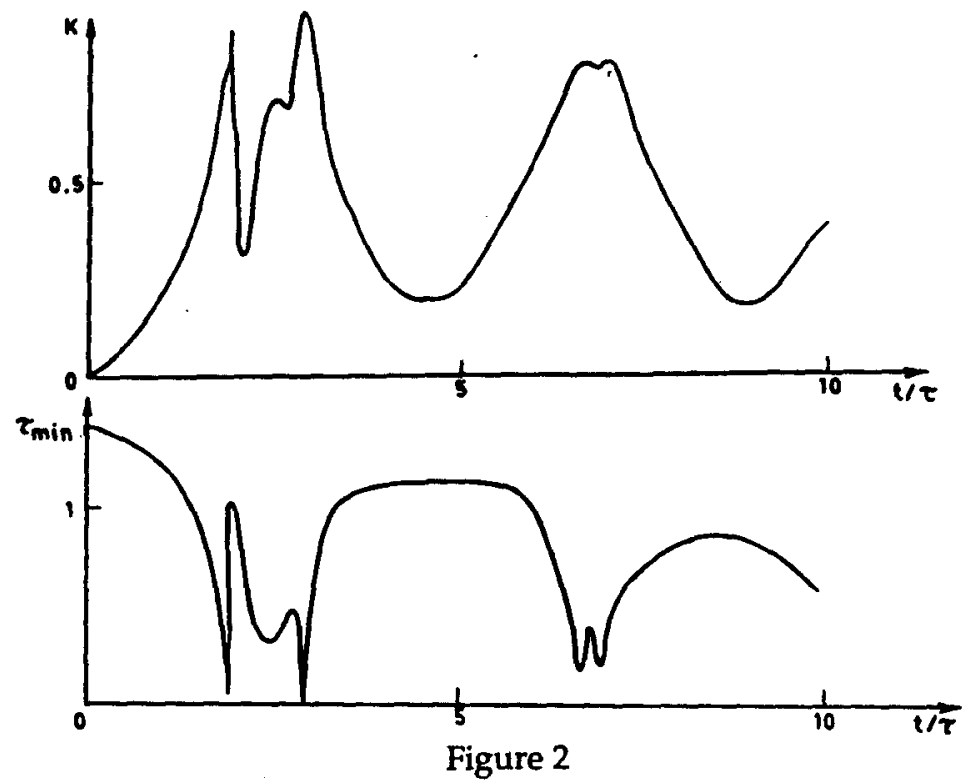




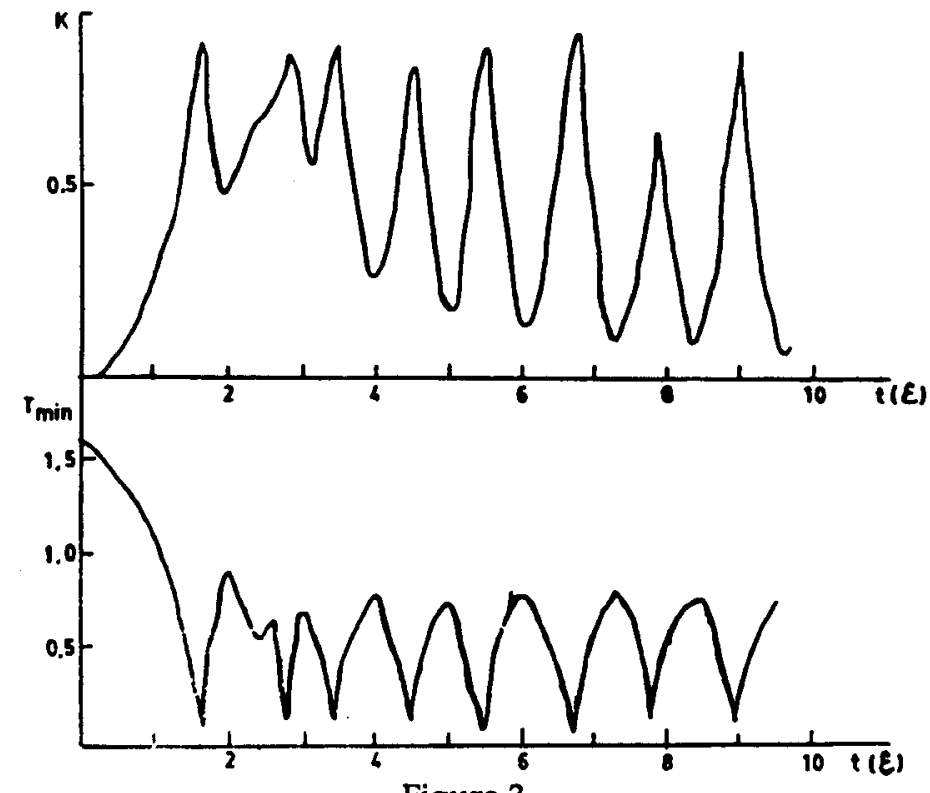

Figure 3

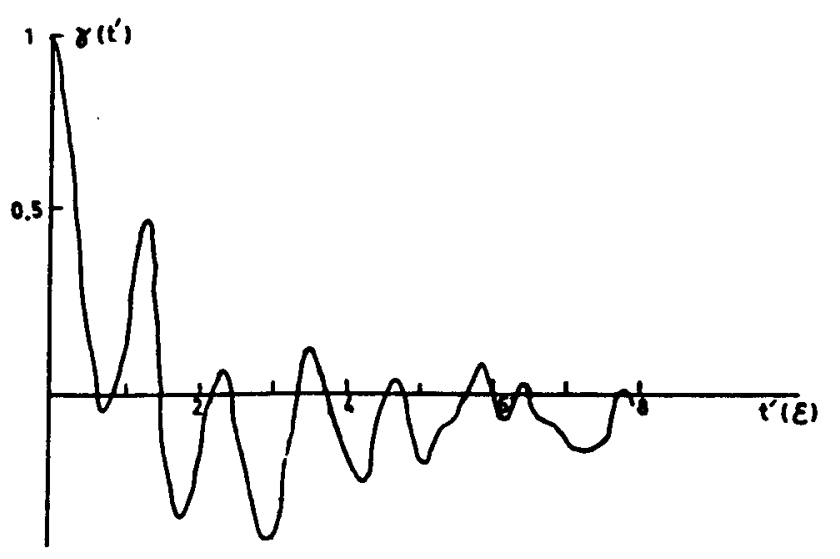

Figure 4 


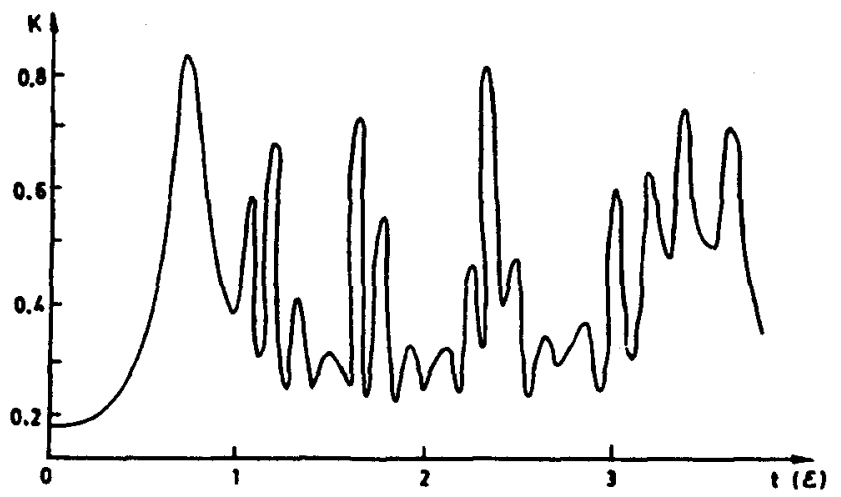

Figure 5

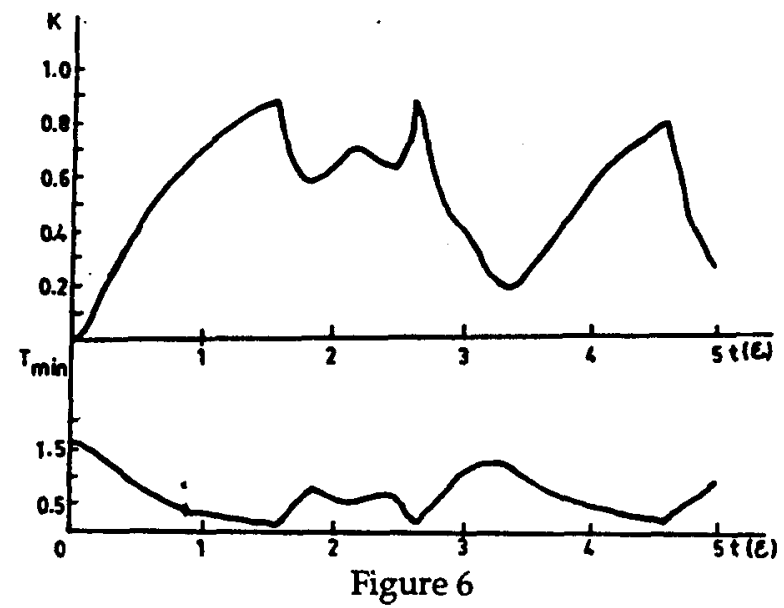




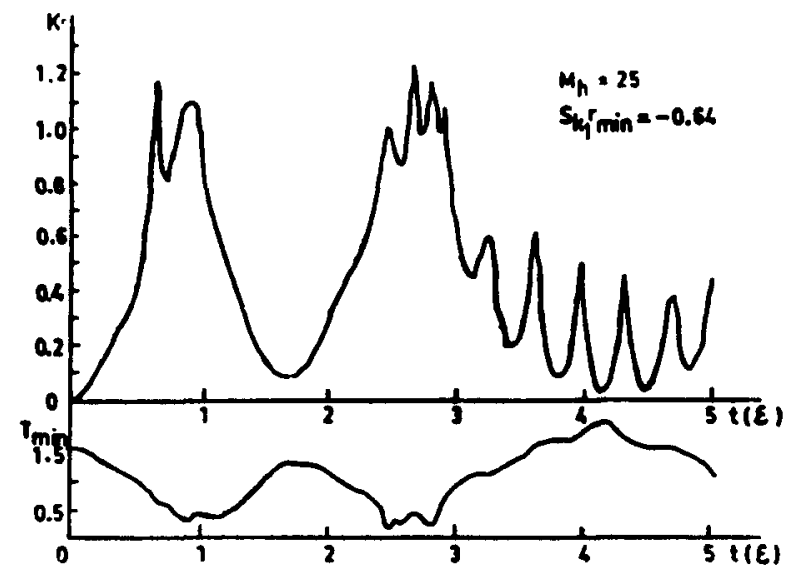

Figure 7

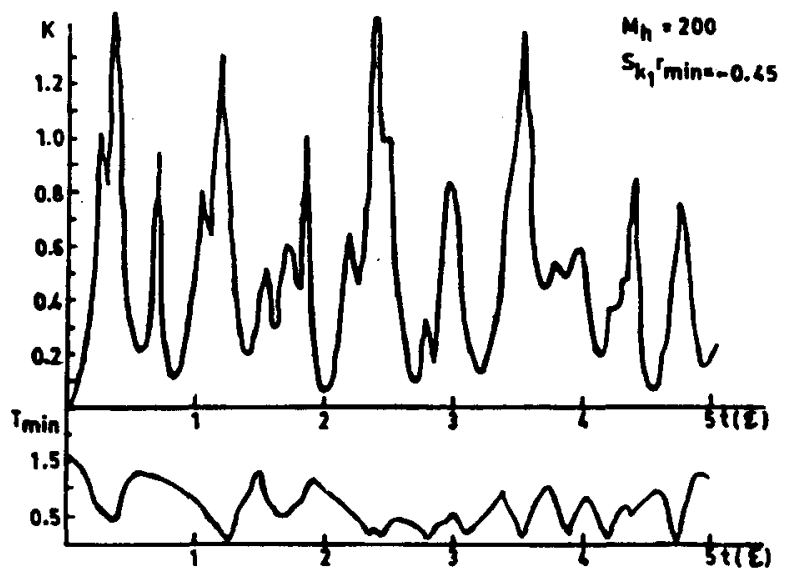

Figure 8 


\section{CONCLUSIONS}

By the computer simulations we have studied a behaviour of the virial coefficient $k(t)$ versus time for the triples and quintets of galaxies. In a number of cases we have some quasiperiodical oscillations $k(t)$ connected with a presence of the temporary binary subsystems. The strong negative correlation between $k$ and minimum separation $r_{m i n}$ takes place. The influence of hidden mass $M_{h}$ to the dynamics of $k(t)$ has been studied. If $M_{h}$ increases then a degree of stochasticity of $k(t)$ increases and the correlation between $k$ and $r_{m i n}$ decreases. The amplitude of oscillations $k(t)$ increases to 0.97 that may lead to the uncertainties in the viriai estimations of masses about one-two orders.

\section{REFERENCES}

1 Ano sova, J.P., Chernin, A.D., Ivanov, A.V ., Kiseleva, L.G. and Orlov, V.V. (1990), IAU Colloquium 132, Abstract.

2 Ano sova, J.P., Ki seleva, L.G., Orlov, V.V., Chernin,A. D., (1 989 ), Astrophys. Space Sci., 158,19.

3 Heisler, J., Tremaine, S. and Bahcall, J.N. (1985), Astrophys.J., 298,8 .

4 Roo s, N. and Norman, G.A. (1979), Astron. Astrophys.76, 75 . 